\title{
Sarcopenia diagnostic criteria update by EWGSOP: what has been changed?
}

\author{
Hidenori Arai ${ }^{1}[\mathbb{C}$
}

Received: 21 September 2018 / Accepted: 27 September 2018

(c) European Geriatric Medicine Society 2018

Sarcopenia is a global health care issue. Since Rosenberg coined the notion of "sarcopenia" in 1989, a large number of studies have been published to address the role of sarcopenia in community-dwelling older people and various clinical settings. In 2010, an epoch-making paper was published by the European Working Group on Sarcopenia in Older People (EWGSOP), in which they proposed the operational diagnostic criteria for sarcopenia that aimed to identify and care people with sarcopenia [1]. In the diagnostic criteria, measurements of grip strength and gait speed along with appendicular muscle mass were required for the diagnosis of sarcopenia. Since then, several groups including the Asian Working Group for Sarcopenia (AWGS) have published similar diagnostic algorithms for sarcopenia [2-4]. We basically followed the approach of EWGSOP and tried to propose the cut-off points for Asian people. The EWGSOP paper has been greatly contributed to the sarcopenia research and their paper has been cited more than 1000 times. Recently, we have published the sarcopenia clinical guidelines in Japan for the first time in the world [5-8]. By conducting systematic review, we found that more and more evidence is required to fill the gap of knowledge in various clinical settings and to provide the data on how to prevent and treat sarcopenia. Introduction of new pharmacological agents is expected in addition to nutritional and exercise interventions.

8 years after the first publication, EWGSOP has published the new consensus of sarcopenia diagnosis to update the original definition, in which they tried to reflect scientific and clinical evidence that has been built so far [9]. In the updated consensus paper on sarcopenia, EWGSOP focuses on low muscle strength as a key characteristic of sarcopenia,

Hidenori Arai is associate editor for Sarcopenia and Frailty of European Geriatric Medicine.

Hidenori Arai

harai@ncgg.go.jp

1 National Center for Geriatrics and Gerontology, Obu, Japan uses low muscle quantity and quality to confirm the sarcopenia diagnosis, and defines severe sarcopenia when poor physical performance is present. They also provide cut-off points for measurements of variables for the diagnosis of sarcopenia. The revised diagnostic criteria are quite well appreciated, considering the age-related changes of skeletal muscle and availability of measurements in various settings. In the aging process, skeletal muscle starts to decline, followed by the decline of power, strength and gait speed. Additionally, grip strength has been shown to better predict adverse health outcomes than skeletal muscle mass [10]. Therefore, it would be reasonable to use grip strength as the primary measure to make sarcopenia diagnosis. However, there are several issues that need to be discussed in more details. The first one is the screening by SARC-F. Although this is a good screening tool for sarcopenia, its low sensitivity may not be appropriate for the screening purpose [11]. The second is which outcome measures are the most appropriate for sarcopenia interventions. Although this topic might be out of scope of this article, it would be better to address this for the future sarcopenia intervention studies. The third is about the diagnosis of secondary sarcopenia in clinical settings. Although we have several outcome studies in the community settings, few studies have been done to address how we should make a diagnosis of sarcopenia in clinical settings, such as patients with chronic renal failure, heart failure, rheumatoid arthritis and so on. More evidence is needed to address this issue.

In spite of these issues, EWGSOP2 will encourage more research in the field of sarcopenia for the prevention of adverse health outcomes that will produce a heavy burden for our societies.

\section{Compliance with ethical standards}

Conflict of interest The corresponding author states that there is no conflict of interest. 
Ethical approval This study does not contain any studies with human participants performed by the author.

Informed consent For this type of study no formal consent is required.

\section{References}

1. Cruz-Jentoft AJ, Baeyens JP, Bauer JM, Boirie Y, Cederholm T, Landi F et al (2010) Sarcopenia: European consensus on definition and diagnosis: report of the European Working Group on Sarcopenia in Older People. Age Ageing 39:412-423

2. Morley JE, Abbatecola AM, Argiles JM, Baracos V, Bauer J, Bhasin S et al (2011) Sarcopenia with limited mobility: an international consensus. J Am Med Dir Assoc 12:403-409

3. Studenski SA, Peters KW, Alley DE, Cawthon PM, McLean RR, Harris TB et al (2014) The FNIH sarcopenia project: rationale, study description, conference recommendations, and final estimates. J Gerontol A Biol Sci Med Sci 69:547-558

4. Chen LK, Liu LK, Woo J, Assantachai P, Auyeung TW, Bahyah KS et al (2014) Sarcopenia in Asia: consensus report of the Asian Working Group for Sarcopenia. J Am Med Dir Assoc 15:95-101
5. Akishita M, Kozaki K, Iijima K, Tanaka T, Shibasaki K, Ogawa S, et al (2018) Chapter 1 definitions and diagnosis of sarcopenia. Geriatr Gerontol Int 18(Suppl 1):7-12

6. Shimokata H, Shimada H, Satake S, Endo N, Shibasaki K, Ogawa S, et al (2018) Chapter 2 epidemiology of sarcopenia. Geriatr Gerontol Int 18(Suppl 1):13-22

7. Kuzuya M, Sugimoto K, Suzuki T, Watanabe Y, Kamibayashi K, Kurihara T, et al (2018) Chapter 3 prevention of sarcopenia. Geriatr Gerontol Int 18(Suppl 1):23-27

8. Arai H, Wakabayashi H, Yoshimura Y, Yamada M, Kim H, Harada A (2018) Chapter 4 treatment of sarcopenia. Geriatr Gerontol Int 18(Suppl 1):28-44

9. Cruz-Jentoft AJ, Bahat G, Bauer J, Boirie Y, Cederholm T, Cooper C, et al (2018) Sarcopenia: revised European consensus on definition and diagnosis. Age Ageing (in press)

10. Newman AB, Kupelian V, Visser M, Simonsick EM, Goodpaster $\mathrm{BH}$, Kritchevsky SB et al (2006) Strength, but not muscle mass, is associated with mortality in the health, aging and body composition study cohort. J Gerontol A Biol Sci Med Sci 61:72-77

11. Woo J, Leung J, Morley JE (2014) Validating the SARC-F: a suitable community screening tool for sarcopenia? J Am Med Dir Assoc 15:630-634 